\title{
BiTR: Built-in Tamper Resilience
}

\author{
Seung Geol Choi ${ }^{1}$, Aggelos Kiayias ${ }^{2, \star}$, and Tal Malkin ${ }^{3, \star \star}$ \\ 1 University of Maryland \\ sgchoi@cs. umd. edu \\ ${ }^{2}$ University of Connecticut \\ aggelos@cse.uconn.edu \\ 3 Columbia University \\ tal@cs.columbia.edu
}

\begin{abstract}
The assumption of the availability of tamper-proof hardware tokens has been used extensively in the design of cryptographic primitives. For example, Katz (Eurocrypt 2007) suggests them as an alternative to other setup assumptions, towards achieving general UC-secure multi-party computation. On the other hand, a lot of recent research has focused on protecting security of various cryptographic primitives against physical attacks such as leakage and tampering.

In this paper we put forward the notion of Built-in Tamper Resilience (BiTR) for cryptographic protocols, capturing the idea that the protocol that is encapsulated in a hardware token is designed in such a way so that tampering gives no advantage to an adversary. Our definition is within the UC model, and can be viewed as unifying and extending several prior related works. We provide a composition theorem for BiTR security of protocols, impossibility results, as well as several BiTR constructions for specific cryptographic protocols or tampering function classes. In particular, we achieve general UC-secure computation based on a hardware token that may be susceptible to affine tampering attacks. We also prove that two existing identification and signature schemes (by Schnorr and Okamoto, respecitively) are already BiTR against affine attacks (without requiring any modification or endcoding). We next observe that non-malleable codes can be used as state encodings to achieve the BiTR property, and show new positive results for deterministic non-malleable encodings for various classes of tampering functions.
\end{abstract}

\section{Introduction}

Security Against Physical Attacks. Traditionally, cryptographic schemes have been analyzed assuming that an adversary has only black-box access to the underlying functionality, and no way to manipulate the internal state. For example, traditional security definitions for encryption schemes address an adversary who is given the public key but not the private key - and tries to guess something about the plaintext of a challenge ciphertext, by applying some black-box attack (e.g., CPA or CCA). In practical situations, however, an adversary can often do more. For example, when using small

\footnotetext{
* Supported in part by NSF grants 0447808, 0831304, and 0831306.

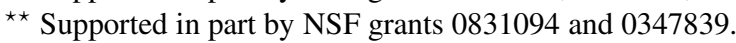


portable devices such as smart-cards or mobile-phones, an adversary can take hold of the device and apply a battery of attacks. One class of attacks are those that try to recover information via side channels such as power consumption [29], electromagnetic radiation [38], and timing [11]. To address these attacks, starting with the work of [2733] there has been a surge of recent research activity on leakage-resilient cryptographic schemes. For example, refer to [41|37|1|22 [10|19|32 9 31] and the references therein. The present work addresses tampering attacks, where an adversary can modify the secret data by applying various physical attacks (c.f., [2]8|7|40|4]). Currently, there are only a few results in this area [23 26 21$]$.

Hardware Tokens. As discussed above, cryptographic primitives have traditionally been assumed to be tamper (and leakage) proof. In the context of larger cryptographic protocols, there have been many works that (implicitly or explicitly) used secure hardware as a tool to achieve security goals that could not be achieved otherwise. The work most relevant to ours is that of Katz [28], who suggests to use tamper-proof hardware tokens to achieve UC-secure [12] commitments. This allows achieving general feasibility results for UC-secure well-formed multi-party computation, where the parties, without any other setup assumptions, send each other tamper-proof hardware tokens implementing specific two-party protocols. There were several follow-up works such as [34 $\left.\begin{array}{ll|l|l|l|l|l|l|l}36 & 18 & 25 & 30 & 24 & 20\end{array}\right]$, all of which assume a token that is tamper proof.

Given the wide applicability of tamper-proof tokens on one hand, and the reality of tampering attacks on the other, we ask the following natural question:

Can we relax the tamper-proof assumption, and get security using tamperable hardware tokens?

Clearly, for the most general interpretation of this question, the answer is typically negative. For example, if the result of [28] was achievable with arbitrarily-tamperable hardware token, that would give general UC-secure protocols in the "plain" model, which is known to be impossible [13]. In this work we address the above question in settings where the class of possible tampering functions and the class of protocols we wish to put in a token and protect are restricted.

\subsection{Our Contributions}

BiTR Definition. We provide a definition of Built-in Tamper Resilience (BiTR) for two party cryptographic protocols, capturing the idea that the protocol can be encapsulated in a hardware token, whose state may be tamperable. Our definition is very general, compatible with the UC setting [12], and implies that any BiTR protocol can be used as a hardware token within larger UC-protocols. Our definition may be viewed as unifying and generalizing previous definitions [23 2621] and bringing them to the UC setting.

BiTR is a property of a cryptographic protocol $M$, which roughly says the following. Any adversary that is able to apply tampering functions from the class $\mathcal{T}$ on a token running $M$, can be simulated by an adversary that has no tampering capability, independently of the environment in which the tokens may be deployed.

The strongest result one would ideally want is a general compiler that takes an arbitrary protocol and transforms it to an equivalent protocol that is BiTR against arbitrary 
tampering functions, without having to encode the state into a larger one, and without requiring any additional randomness 1 Since such a strong result is clearly impossible, we provide several specific results that trade off these parameters (see below), as well as the following composition theorem.

BiTR Composition. As BiTR is a protocol centric property, the natural question that arises is whether it is preserved under composition. A useful result for a general theory of BiTR cryptography would be a general composition theorem which allows combining a BiTR protocol calling a subroutine and a BiTR implementation of that subroutine into one overall BiTR protocol. To this end, we characterize BiTR composition of protocols by introducing the notion of modular-BiTR which captures the property of being BiTR in the context of a larger protocol. We then prove that the property of modular-BiTR is necessary and sufficient for construction of composite BiTR protocols. At the same time we also derive a negative result, namely that modular-BiTR protocols that preserve the BiTR property in any possible context (something we term universal-BiTR) are unattainable assuming the existence of one-way functions, at least for non-trivial protocols. These results thus settle the question of BiTR composability.

BiTR Constructions without State Encoding. We describe results for BiTR primitives that require no state encodings. It may come as a surprise that it is possible to prove a cryptographic protocol BiTR without any encoding and thus without any validation of the secret protocol state whatsoever. This stems from the power of our definitional framework for BiTR and the fact that it is can be achieved for specially selected and designed protocols and classes of tampering functions. We define the class $\mathcal{T}_{\text {aff }}=\left\{f_{a, b} \mid a \in \mathbb{Z}_{q}^{*}, b \in \mathbb{Z}_{q}, f_{a, b}(v):=a v+b \bmod q\right\}$. That is, the adversary may apply a modular affine function of his choice to tamper the state. Affine tampering is an interesting class to consider as it has as special cases multiplication (e.g., shifting which may be the result of tampering shift-register based memory storage), or addition (which may be result of bit flipping tampering).

We prove three protocols BiTR with respect to this class, where the tamper resilience is really "built-in" in the sense that no modification of the protocol or encoding of the state are necessary. The first one is Schnorr's identification (two-round) protocol [39]. The second is Okamoto's signature scheme [35]. Both protocols are interesting on their own (e.g., previous work [23] focused mostly on signature schemes), but the latter is also useful for the third protocol we prove affine-BiTR, described next.

UC-Secure Computation from tamperable tokens. Katz's approach [28] for building UC-secure computation using hardware tokens allows a natural generalization that involves a commitment scheme with a special property, we call a dual-mode parameter generation $(D P G)$ - depending on the mode of the parameter, the commitment scheme is either statistically hiding or a trapdoor commitment. We then observe that any DPGcommitment is sufficient for providing UC-secure multi-party computation assuming tamper proof tokens. Following this track, we present a new DPG-commitment scheme

\footnotetext{
${ }^{1}$ If an encoding $\psi$ of the state is required, it is desirable that it is deterministic (randomness may not be available in some systems or expensive to generate), and that it has as high rate as possible. Ideally, an existing scheme can be proven BiTR as-is, without any state encoding at all.
} 
that is BiTR against affine tampering functions, that relies on discrete-log based primitives including the digital signature scheme of Okamoto [35]. Thus, we obtain UCsecure general computation using hardware tokens tamperable with affine functions.

BiTR Constructions with State Encoding. We next discuss how one can take advantage of state consistency checks to design BiTR protocols. We observe first that nonmalleable codes, introduced by Dziembowski, Pietrzak and Wichs [21] can be used as an encoding for proving the BiTR property of protocols. This gives rise to the problem of constructing such codes. Existing constructions [21] utilize randomness in calculating the encoding; we provide new constructions for such encodings focusing on purely deterministic constructions. In fact, when the protocol uses no randomness (e.g., a deterministic signing algorithm) or a finite amount of randomness (e.g., a prover in the resettable zero-knowledge [14] setting), by using deterministic encodings the token may dispense with the need of random number generation.

Our design approach takes advantage of a generalization of non-malleable encodings (called $\delta$-non-malleable), and we show how they can be constructible for any given set of tampering functions (as long as they exist). Although inefficient for general tampering functions, the construction becomes useful if each function in the class $\mathcal{T}$ works independently on small blocks (of logarithmic size). In this case, we show that a nonmalleable code for the overall state can be constructed efficiently by first applying ReedSolomon code to the overall state and then applying $\delta$-non-malleable codes for small blocks to the resulting codeword. We stress that this construction is intended as a feasibility result.

\subsection{Related Work}

We briefly describe the most relevant previous works addressing protection against tampering. We note that none of these works had addressed tampering in the context of UC-secure protocols.

Gennaro et al. [23] considered a device with two separate components: one is tamperproof yet readable (circuitry), and the other is tamperable yet read-proof (memory). They defined algorithmic tamper-proof (ATP) security and explored its possibility for signature and decryption devices. Their definition of ATP security was given only for the specific tasks of signature and encryption. In contrast, our definition is simulation based, independent of the correctness or security objectives of the protocol, and we consider general two-party protocols (and the implications in the UC framework [12[28]).

Ishai et al. [26] considered an adversary who can tamper with the wires of a circuit. They showed a general compiler that outputs a self-destructing circuit that withstands such a tampering adversary. Considering that memory corresponds to a subset of the wires associated with the state in their model, the model seems stronger than ours (as we consider only the state, not the computation circuit). However, the tampering attack they considered is very limited: it modifies a bounded subset of the wires between each invocation, which corresponds to tampering memory only partially.

Dziembowski et al. [21] introduced the notion of non-malleable codes and tamper simulatability to address similar concerns as the present work. A distinguishing feature of BiTR security from their approach is that BiTR is protocol-centric. As such, it allows 
arguing about tamper resilience by taking advantage of specific protocol design features that enable BiTR even without any encodings. Moreover, the positive results of [21] require the introduction of additional circuitry or a randomness device; this may be infeasible, uneconomical or even unsafe in practice - it could be introducing new pathways for attacks. In contrast, our positive results do not require state encodings or when they do, they do not rely on randomness.

Bellare and Kohno defined security against related key attacks (RKA) for block ciphers [6], and there has been follow-up work [53] (see also the references therein). Roughly speaking, RKA-security as it applies to PRFs and encryption is a strengthening of the security definition of the underlying primitive (be it indistinguishability from random functions or semantic security). RKA-security was only shown against tampering that included addition or multiplication (but not both simultaneously). In fact, RKA-security for PRFs as defined in [5] is different from BiTR when applied to PRFs. A BiTR PRF is not necessarily RKA-secure since the BiTR simulator is allowed to take some liberties that would violate key independence under tampering as required by RKA-security. We do not pursue these relationships further here formally as it is our intention to capture BiTR in a weakest possible sense and investigate how it captures naturally in a simulation-based fashion the concept of tamper resilience for any cryptographic primitive.

\section{BiTR Definitions}

BiTR Protocols. Katz [28] modeled usage of a tamper-proof hardware token as an ideal functionality $\mathcal{F}_{\text {wrap }}$ in the UC framework. Here, we slightly modify the functionality so that it is parameterized by an interactive Turing machine (ITM) $M$ for a two-party protoco (see Fig. 1). The modification does not change the essence of the wrapper functionality; it merely binds honest parties to the use of a specific embedded program. Corrupted parties may embed an arbitrary program in the token by invoking Forge. We also define a new functionality $\mathcal{F}_{\text {twrap }}$ similar to $\mathcal{F}_{\text {wrap }}$ but with tampering allowed. Let $\mathcal{T}$ be a collection of (randomized) functions. Let $\psi=(E, D)$ be an encoding scheme 3 . The essential difference between $\mathcal{F}_{\text {twrap }}$ and $\mathcal{F}_{\text {wrap }}$ is the ability of the adversary to tamper with the internal state of the hardware token - a function drawn from $\mathcal{T}$ is applied on the internal state of the hardware token. This (weaker) ideal functionality notion is fundamental for the definition of BiTR that comes next.

We define a security notion for a protocol $M$, called Built-in Tamper Resilience (BiTR), which essentially requires that $\mathcal{F}_{\text {twrap }}(M)$ is interchangeable with $\mathcal{F}_{\text {wrap }}(M)$. We adopt the notations in the UC framework given by Canetti [12].

Definition 1 (BiTR protocol). The protocol $M$ is $(\mathcal{T}, \psi)$-BiTR iffor any PPT $\mathcal{A}$, there exists a PPT $\mathcal{S}$ such that for any non-uniform PPT $\mathcal{Z}$,

$$
\operatorname{IDEAL}_{\mathcal{F}_{\text {twrap }}(M, \mathcal{T}, \psi), \mathcal{A}, \mathcal{Z}} \approx \operatorname{IDEAL}_{\mathcal{F}_{\text {wrap }}(M), \mathcal{S}, \mathcal{Z}},
$$

where $\approx$ denotes computational indistinguishability.

\footnotetext{
${ }^{2}$ We will interchangeably use protocols and ITMs, if there is no confusion.

${ }^{3}$ We will sometimes omit $\psi$ from $\mathcal{F}_{\text {twrap }}$ when it is obvious from the context.
} 
$\mathcal{F}_{\text {wrap }}(M)$ is parameterized by a polynomial $p$ and a security parameter $k$.

Create: Upon receiving $\left\langle\right.$ Create, sid, $\left.P, P^{\prime}, m s g\right\rangle$ from party $P$ : Let $m s g^{\prime}=$ (Initialize, $m s g)$. Run $M\left(m s g^{\prime}\right)$ for at most $p(k)$ steps. Let out be the response of $M$ (set out to $\perp$ if $M$ does not respond). Let $s^{\prime}$ be the updated state of $M$. Send Initialized, sid, $P^{\prime}$, out $\rangle$ to $P$, and $\left\langle\right.$ Create, sid, $\left.P, P^{\prime}, 1^{\left|s^{\prime}\right|}\right\rangle$ to $P^{\prime}$ and the adversary. If there is no record $\left(P, P^{\prime}, *, *\right)$, then store $\left(P, P^{\prime}, M, s^{\prime}\right)$.

Forge: Upon receiving $\left\langle\right.$ Forge, sid, $\left.P, P^{\prime}, M^{\prime}, s\right\rangle$ from the adversary, if $P$ is not corrupted, do nothing. Otherwise, send $\left\langle\right.$ Create, sid, $\left.P, P^{\prime}, 1^{|s|}\right\rangle$ to $P^{\prime}$. If there is no record $\left(P, P^{\prime}, *, *\right)$, then store $\left(P, P^{\prime}, M^{\prime}, s\right)$.

Run: Upon receiving $\langle$ Run, sid, $P, m s g\rangle$ from party $P^{\prime}$, find a record $\left(P, P^{\prime}, K, s\right)$. If there is no such record, do nothing. Otherwise, do:

1. Run $K(\mathrm{msg} ; \mathrm{s})$ for at most $p(k)$ steps. Let out be the response of $K$ (set out to $\perp$ if $K$ does not respond). Let $s^{\prime}$ be the updated state of $K$. Send (sid, $P$, out) to $P^{\prime}$.

2. Update the record with $\left(P, P^{\prime}, K, s^{\prime}\right)$.

$\mathcal{F}_{\text {twrap }}(M, \mathcal{T}, \psi)$ is also parameterized by $p$ and $k$ (and $\psi=(E, D)$ is an encoding scheme).

Create: As in $\mathcal{F}_{\text {wrap }}(M)$ with the only change that state $s^{\prime}$ is stored as $E\left(s^{\prime}\right)$ in memory.

Forge: As in $\mathcal{F}_{\text {wrap }}(M)$.

Run: Upon receiving $\langle$ Run, sid, $P, m s g\rangle$ from party $P^{\prime}$, find a record $\left(P, P^{\prime}, K, \tilde{s}\right)$. If there is no such record, do nothing. Otherwise, do:

1. (Tampering) If $P^{\prime}$ is corrupted and a record $\left\langle\right.$ sid, $\left.P, P^{\prime}, \tau\right\rangle$ exists, set $\tilde{s}=\tau(\tilde{s})$ and erase the record.

2. (Decoding) If $P$ is corrupted, set $s=\tilde{s}$; otherwise, set $s=D(\tilde{s})$. If $s=\perp$, send $($ sid $, P, \perp)$ to $P^{\prime}$ and stop.

3. Run $K(\mathrm{msg} ; \mathrm{s})$ for at most $p(k)$ steps. Let out be the response of $K$ (set out to $\perp$ if $K$ does not respond). Let $s^{\prime}$ be the updated state of $K$. Send $\left(\right.$ sid, $P$, out) to $P^{\prime}$.

4. (Encoding) If $P$ is corrupted, set $\tilde{s}=s^{\prime}$; otherwise set $\tilde{s}=E\left(s^{\prime}\right)$. Update the record with $\left(P, P^{\prime}, K, \tilde{s}\right)$.

Tamper: Upon receiving $\left\langle\right.$ Tamper, sid, $\left.P, P^{\prime}, \tau\right\rangle$ from the adversary $\mathcal{A}$, if $P^{\prime}$ is not corrupted or $\tau \notin \mathcal{T}$, do nothing. Otherwise make a record (sid, $\left.P, P^{\prime}, \tau\right)$ (erasing any previous record of the same form).

Fig. 1. Ideal functionalities $\mathcal{F}_{\text {wrap }}(M)$ and $\mathcal{F}_{\text {twrap }}(M, \mathcal{T}, \psi)$

In case $\psi=$ (id, id) (i.e., identify functions), we simply write $\mathcal{T}$-BiTR. Note that this definition is given through the ideal model, which implies (by the standard UC theorem) that whenever a tamper-proof token wrapping $M$ can be used, it can be replaced by a $\mathcal{T}$-tamperable token wrapping $M 4$ As a trivial example, every protocol is $\{$ id $\}$-BiTR.

We note that the above definition is intended to capture in the weakest possible sense the fact that a protocol is tamper resilient within an arbitrary environment. A feature of the definition is that there is no restriction in the way the simulator accesses the underlying primitive (as long as no tampering is allowed). This enables, e.g., a signature to be called BiTR even if simulating tampered signatures requires untampered signatures on different chosen messages, or even on a larger number of chosen messages. We believe

\footnotetext{
${ }^{4}$ One could also consider a definition that requires this in the context of a specific UC-protocol. We believe our stronger definition, which holds for any UC-protocol using a token with $M$, is the right definition for built-in tamper resilience.
} 
that this is the correct requirement for the definition to capture that "if the underlying primitive is secure without tampering, it is secure also with tampering" (in the signature example, security is unforgeability against any polynomial time chosen message attack). Nonetheless, it can be arguably even better to achieve BiTR security through a "tighter" simulation, where the BiTR simulator is somehow restricted to behave in a manner that is closer to the way $\mathcal{A}$ operates (except for tampering of course) or possibly even more restricted. For instance, one may restrict the number of times the token is accessed by the simulator to be upper bounded by the number of times $\mathcal{A}$ accesses the token. In fact all our positive results do satisfy this desired additional tighter simulation property. Taking this logic even further, one may even require that once tampering occurs the BiTR simulator can complete the simulation without accessing the token at all — effectively suggesting that tampering trivializes the token and makes it entirely simulatable. We believe that the ability of BiTR to be readily extended to capture such more powerful scenarios highlights the robustness of our notion and, even though these scenarios are not further pursued here, the present work provides the right basis for such upcoming investigations.

\subsection{Composition of BiTR ITMs}

It is natural to ask if a modular design approach applies to BiTR protocols. To investigate this question we need first to consider how to define the BiTR property in a setting where protocols are allowed to call subroutines.

Consider an ITM $M_{2}$ and another ITM $M_{1}$ that calls $M_{2}$ as a subroutine. We denote by $\left(M_{1} ; M_{2}\right)$ the compound ITM. The internal state of $\left(M_{1} ; M_{2}\right)$ is represented by the concatenation of the two states $s_{1} \| s_{2}$ where $s_{1}$ and $s_{2}$ are the states of $M_{1}$ and $M_{2}$ at a certain moment of the runtime respectively. Let $\mathcal{F}_{\text {twrap }}\left(M_{1} ; M_{2}, \mathcal{T}_{1} \times \mathcal{T}_{2}, \psi_{1} \times \psi_{2}\right)$ denote an ideal functionality that permits tampering with functions from $\mathcal{T}_{1}$ for the state of $M_{1}$ and from $\mathcal{T}_{2}$ for the state of $M_{2}$ while the states are encoded with $\psi_{1}$ and $\psi_{2}$ respectively. We can also consider a sequence of ITMs that call each other successively $\bar{M}=\left(M_{1} ; \ldots ; M_{n}\right)$. We next generalize the BiTR notion for an ITM $M_{i}$ employed in the context of $\bar{M}$ in a straightforward manner.

Definition 2 (modular BiTR protocol). Given $\bar{M}=\left(M_{1} ; \ldots ; M_{n}\right), \overline{\mathcal{T}}=\mathcal{T}_{1} \times \ldots \times$ $\mathcal{T}_{n}$, and $\bar{\psi}=\psi_{1} \times \ldots \times \psi_{n}$, for some $i \in[n]$, we say that $M_{i}$ is modular $-\left(\mathcal{T}_{i}, \psi_{i}\right)-$ BiTR with respect to $\bar{M}, \overline{\mathcal{T}}$ and $\bar{\psi}$ if for any PPT $\mathcal{A}$ there exists a PPT $\mathcal{S}$ such that for any non-uniform PPT $\mathcal{Z}$,

$$
\begin{aligned}
& \operatorname{IDEAL}_{\mathcal{F}_{\text {twrap }}\left(\bar{M}, \overline{\mathcal{T}_{i}}, \bar{\psi}\right), \mathcal{A}, \mathcal{Z}} \approx \operatorname{IDEAL}_{\mathcal{F}_{\text {twrap }}\left(\bar{M}, \overline{\mathcal{T}_{i+1}}, \bar{\psi}\right), \mathcal{S}, \mathcal{Z}}, \\
& \text { where } \overline{T_{i}}=\{\mathrm{id}\} \times \ldots \times\{\mathrm{id}\} \times \mathcal{T}_{i} \times \ldots \times \mathcal{T}_{n} .
\end{aligned}
$$

Roughly speaking, this definition requires that whatever the adversary can do by tampering $M_{i}$ with $\mathcal{T}_{i}$ (on the left-hand side) should be also done without (on the right-hand side) in the context of $\bar{M}, \overline{\mathcal{T}}, \bar{\psi}$. For simplicity, if $\bar{M}, \overline{\mathcal{T}}, \bar{\psi}$ are clear from the context, we will omit a reference to it and call an ITM $M_{i} \operatorname{simply}$ modular- $\left(\mathcal{T}_{i}, \psi_{i}\right)$-BiTR.

The composition theorem below confirms that each ITM being modular BiTR is a necessary and sufficient condition for the overall compound ITM being BiTR. 
Theorem 1 (BiTR Composition Theorem). Consider protocols $M_{1}, \ldots, M_{n}$ with $\bar{M}=\left(M_{1}, \ldots, M_{n}\right)$ and $\mathcal{T}=\mathcal{T}_{1} \times \ldots \times \mathcal{T}_{n}$, and $\psi=\psi_{1} \times \ldots \times \psi_{n}$. It holds that $M_{i}$ is modular- $\left(\mathcal{T}_{i}, \psi_{i}\right)$-BiTR for $i=1, \ldots, n$, with respect to $\bar{M}, \overline{\mathcal{T}}, \bar{\psi}$ if and only if $\left(M_{1} ; \ldots ; M_{n}\right)$ is $(\mathcal{T}, \psi)$-BiTR.

A natural task that arises next is to understand the modular-BiTR notion.

Context Sensitivity of Modular-BiTR Security. The modular-BiTR definition is context-sensitive; an ITM may be modular BiTR in some contexts but not in others, in particular depending on the overall compound token $\bar{M}$. This naturally begs a question whether there is a modular-BiTR ITM that is insensitive to the context. In this way, akin to a universally composable protocol, a universally BiTR ITM could be used modularly together with any other ITM and still retain its BiTR property. To capture this we formalize universal-BiTR security below, as well as a weaker variant of it that is called universal-BiTR parent which applies only to ITMs used as the parent in a sequence of ITMs.

Definition 3 (universal BiTR). If an ITM $M$ is modular- $(\mathcal{T}, \psi)$-BiTR with respect to any possible $\bar{M}, \overline{\mathcal{T}}, \bar{\psi}$ then we call $M$ universal- $(\mathcal{T}, \psi)$-BiTR. If $M$ is modular- $(\mathcal{T}, \psi)$ BiTR whenever $M$ is used as the parent ITM then we call it universal- $(\mathcal{T}, \psi)$-BiTR parent.

Not very surprisingly (and in a parallel to the case of UC protocols) this property is very difficult to achieve. In fact, we show that if one-way functions exist then nontrivial universal-BiTR ITMs do not exist. We first define non-triviality: an ITM $M$ will be called non-trivial if the set of its states can be partitioned into at least two sets $S_{0}, S_{1}$ and there exists a set of inputs $A$ that produce distinct outputs depending when the ITM $M$ is called and its internal state belongs to $S_{0}$ or $S_{1}$. We call the pair of sets a state partition for $M$ and the set $A$ the distinguishing input-set. Note that if an ITM is trivial then for any partition of the set of states $S_{0}, S_{1}$ and any set of inputs $A$, the calling of the ITM $M$ on $A$ produces identical output. This effectively means that the ITM $M$ does not utilize its internal state at all and obviously is BiTR by default. Regarding nontrivial ITMs we next prove that they cannot be $(\mathcal{T}, \psi)$-BiTR for any tampering function $\tau$ that switches the state between the two sets $S_{0}, S_{1}$, i.e., $\tau\left(S_{0}\right) \subseteq S_{1}, \tau\left(S_{1}\right) \subseteq S_{0}$. We call such tampering function state-switching for the ITM $M$. If an encoding $\psi$ is involved, we call $\tau$ state-switching for the encoding $\psi$. We are now ready to prove our negative result.

Theorem 2. Assuming one-way functions exist, there is no non-trivial universal-( $\mathcal{T}, \psi)$ BiTR ITM $M$ such that $\mathcal{T}$ contains a state-switching function for $M$ and the encoding $\psi$.

Roughly speaking, the theorem holds since a parent ITM $M_{1}$ calling $M_{2}$ can make the message exchanges between them quite non-malleable by outputting a signature on these messages. In this context, no non-trivial $M_{2}$ can be modular-BiTR, and thus $M_{2}$ is not universal-BiTR. We note that the above theorem is quite final for the case of universal BiTR ITMs. It leaves only the possibility of proving the universal-BiTR property for trivial ITMs (that by default satisfy the notion) or for sets of functions that 
are not state-switching, i.e., essentially they do not affect the output of $M$ and therefore inconsequential. This state of affairs is not foreign to properties that are supposed to universally compose. Indeed, in the case of UC-security large classes of functionalities are not UC-realizable [15]. To counter this issue, in the UC-setting one may seek setup assumptions to alleviate this problem, but in our setting setup assumptions should be avoided. For this reason, proving the modular-BiTR property within a given context is preferable.

On the other hand, the universal-BiTR parent property turns out to be feasible, and thus this leaves a context insensitive property to be utilized for modular design of BiTR protocols. We in fact take advantage of this, and jumping ahead, the parent ITM in the compound ITM used to achieve general UC-secure MPC in Section 4 satisfies this property and can be composed with any child ITM.

\section{Affine BiTR Protocols without State Encoding}

In this section, we show two protocols (for identification and signatures, respectively) that are BiTR against certain tampering functions, without using any modification or encoding. Specifically, we consider a tampering adversary that can modify the state of the hardware with affine functions. Assuming the state of the hardware is represented by variables of $\mathbb{Z}_{q}$ for some prime $q$, the adversary can choose a tampering $f_{a, b}$ on a variable $v$, which will change $v$ into $f_{a, b}(v)=a v+b \bmod q$. Let $\mathcal{T}_{\text {aff }}=\left\{f_{a, b} \mid a \in\right.$ $\left.\mathbb{Z}_{q}^{*}, b \in \mathbb{Z}_{q}\right\}$ and $\mathcal{T}_{\text {aff }}^{2}=\mathcal{T}_{\text {aff }} \times \mathcal{T}_{\text {aff }}$.

Schnorr Identification [39]. The Schnorr identification is a two-round two-party protocol between a prover and a verifier. The common input is $y=g^{x}$, where $g$ is a generator of a cyclic group of size $q$, and the prover's auxiliary input is $x \in \mathbb{Z}_{q}$. The protocol proceeds as follows:

1. The prover picks a random $t \in \mathbb{Z}_{q}$ and sends $z=g^{t}$ to the verifier.

2. The verifier picks a random $c \in \mathbb{Z}_{q}$ and sends $c$ to the prover, which in turn computes $s=c x+t \bmod q$ and sends $s$ to the verifier. The verifier checks if $z y^{c}=g^{s}$.

We consider an ITM $M$ on the prover side wrapped as a hardware token. This ITM is BiTR against affine functions. To see why it is BiTR, suppose that the adversary tampers with the state changing $x$ into $a x+b$ for some $a$ and $b$. In the second round, the BiTR simulator - given $c$, from the adversary, that is supposed to go to $\mathcal{F}_{\text {twrap }}\left(M ; \mathcal{T}_{\text {aff }}\right)$ - has to find out an appropriate $c^{\prime}$ going to $\mathcal{F}_{\text {wrap }}(M)$ such that the simulator, on receiving $s^{\prime}=c^{\prime} x+t$ from $\mathcal{F}_{\text {wrap }}(M)$, can output $c(a x+b)+t$ that would come from $\mathcal{F}_{\text {twrap }}\left(M ; \mathcal{T}_{\text {aff }}\right)$. In summary, given $\left(a, b, c, s^{\prime}\right)$, but not $x$ or $t$, the simulator has to generate a correct output by controlling $c^{\prime}$. It can do so by choosing $c^{\prime}=a c$ and outputting $s^{\prime}+c b$. Note that $s^{\prime}+c b=c(a x+b)+t$.

Signature Scheme due to Okamoto [35]. The digital signature scheme of Okamoto [35] was employed in the context of designing blind signatures. Here we show that it is BiTR against affine functions. We give a brief description next. Let $\left(\mathbb{G}_{1}, \mathbb{G}_{2}\right)$ be a bilinear group as follows: (1) $\mathbb{G}_{1}$ and $\mathbb{G}_{2}$ are two cyclic groups of prime order $q$ possibly with $\mathbb{G}_{1}=\mathbb{G}_{2}$; (2) $h_{1}$ and $h_{2}$ are generators of $\mathbb{G}_{1}$ and $\mathbb{G}_{2}$ respectively; (3) 
$M_{o k a}$ : The description of $\mathbb{G}_{1}, \mathbb{G}_{2}, g_{2}, u_{2}, v_{2}$, and a collision-resistant hashing function $H$ : $\{0,1\}^{n} \rightarrow \mathbb{Z}_{q}^{*}$ are embedded in the program as a public parameter. The state is $x \in \mathbb{Z}_{q}$.

\section{Initialization}

- Upon receiving a message (Initialize), choose $x \in_{R} \mathbb{Z}_{q}$, and $g_{2}, u_{2}, v_{2} \in_{R} G_{2}$ and output $\left(g_{2}, w_{2}, u_{2}, v_{2}\right)$.

\section{Message Handling}

- Upon receiving a message (Sign, $m$ ), Choose random $r, s \in \mathbb{Z}_{q}^{*}$ such that $x+r \neq 0$ $(\bmod q)$. Compute $\sigma=\left(g_{1}^{H(m)} u_{1} v_{1}^{s}\right)^{1 /(x+r)}$ and output $(\sigma, r, s)$.

Fig. 2. Okamoto signature $M_{o k a}$

$\psi$ is an isomorphism from $\mathbb{G}_{2}$ to $\mathbb{G}_{1}$ such that $\psi\left(h_{2}\right)=h_{1} ;$ (4) $e$ is a non-degenerate bilinear map $e: \mathbb{G}_{1} \times \mathbb{G}_{2} \rightarrow \mathbb{G}_{T}$ where $\left|\mathbb{G}_{T}\right|=p, \forall u \in \mathbb{G}_{1} \forall v \in \mathbb{G}_{2} \forall a, b \in \mathbb{Z}$ : $e\left(u^{a}, u^{b}\right)=e(u, v)^{a b}$.

The signature scheme below is secure against a chosen message attack under the Strong Diffie-Hellman assumption [35].

- Key Generation: Randomly select generators $g_{2}, u_{2}, v_{2} \in G_{2}$ and compute $g_{1}=$ $\psi\left(g_{2}\right), u_{1}=\psi\left(u_{2}\right)$, and $v_{1}=\psi\left(v_{2}\right)$. Choose a random $x \in \mathbb{Z}_{q}^{*}$ and compute $w_{2}=g_{2}^{x}$. Verification key is $\left(g_{1}, g_{2}, w_{2}, u_{2}, v_{2}\right)$. Signing key is $x$.

- Signature of a message $m \in \mathbb{Z}_{q}^{*}$ : Choose random $r, s \in \mathbb{Z}_{q}^{*}$. The signature is $(\sigma, r, s)$ where $\sigma=\left(g_{1}^{m} u_{1} v_{1}^{s}\right)^{1 /(x+r)}$ and $x+r \neq 0(\bmod q)$.

- Verification of $(m, \sigma, r, s)$ : Check that $m, r, s, \in \mathbb{Z}_{q}^{*}, \sigma \in \mathbb{G}_{1}, \sigma \neq 1$, and $e\left(\sigma, w_{2} g_{2}^{r}\right)=e\left(g_{1}, g_{2}^{m} u_{2} v_{2}^{s}\right)$.

The signature token is described in Fig. 2. Similarly to the ITM for Schnorr signature scheme, this token can be shown to be BiTR against affine functions.

Theorem 3. ITM $M_{\text {oka }}$ in Fig. 2] is $\mathcal{T}_{\text {aff-BiTR. }}$

\section{UC Secure Computation from Tamperable Tokens}

In this section we examine the problem of achieving UC-secure computation relying on tamperable (rather than tamper-proof) tokens. Our starting point is the result of Katz [28], obtaining a UC commitment scheme (and general UC-secure computation) in the $\mathcal{F}_{\text {wrap }}(M)$-hybrid for an ITM $M$, which unfortunately, is not BiTR. However, we managed to change $M$ so that the modified ITM $M^{\prime}$ is BiTR against affine functions, thus obtaining a UC commitment in the $\mathcal{F}_{\text {twrap }}\left(M^{\prime}\right)$-hybrid. Along the way, we present a generalization of Katz's scheme for building commitment schemes we call commitments with dual-mode parameter generation.

\subsection{Katz's Commitment Scheme and its Generalization.}

Intuitively, the UC-secure commitment scheme given by Katz [28] uses the tamperproof hardware token to give the simulator the advantage over the adversary to force the 
commitment scheme to become extractable (in case the sender is corrupted) or equivocal (in case the receiver is corrupted). In spirit, this idea can be traced to mixed commitment schemes introduced in [17], although the two results differ greatly in techniques.

We abstract the approach of [28] to build UC commitments in Fig. 3] The UC commitment scheme is based on a primitive that we call commitment with dual-mode parameter generation (DPG-commitment for short).

\section{Commitment Phase:}

1. Each of the sender and the receiver calls $\mathcal{F}_{\text {wrap }}(M)$ with a Create message.

2. Each party executes the procedure dual-mode parameter generation with the $\mathcal{F}_{\text {wrap }}(M)$. Let $p S$ be the parameter the receiver obtained, and $p R$ be one the sender obtained. The parameters $p R$ and $p S$ are exchanged.

3. The sender commits to a message $m$ by sending $\left\langle C_{1}, C_{2}, \pi\right\rangle$, where $C_{1}$ is a commitment to $m$ based on the parameter $p S, C_{2}$ is a statistically-binding commitment to $m$, and $\pi$ is WI proof that (1) $C_{1}$ and $C_{2}$ commits to the same message, or (2) $p R$ was generated in the extraction mode.

\section{Opening Phase:}

1. The sender reveals $\left\langle m, \pi^{\prime}\right\rangle$, where $m$ is the committed message, $\pi^{\prime}$ is WI proof that (1) $C_{2}$ commits to $m$, or (2) $p R$ was generated in the extraction mode.

Fig. 3. A UC Commitment that uses a DPG-commitment scheme $\Pi$ with protocol $M$ in the $\mathcal{F}_{\text {wrap }}(M)$-hybrid model.

A DPG-commitment is a commitment scheme whose parameter is generated by an interactive protocol $M$ that is wrapped in a hardware token. Formally we define the following:

Definition 4 (DPG-Commitment scheme). A commitment scheme $\Pi=(\mathrm{Com}$, Decom $)$ that is parameterized by $p$, has a dual mode parameter generation (DPG-commitment) if there are ITMs $M$ and $P$ that form a two party protocol $\langle P, M\rangle$ and have the following properties:

- (Normal mode) For any PPT $P^{*}$, with overwhelming probability, the output of $\left\langle P^{*}, M\right\rangle$ satisfies that if it is not $\perp$ then it contains a parameter $p$ over which the commitment scheme $\Pi$ is unconditionally hiding.

- (Extraction mode) For any $M^{*}$ with the same I/O as $M$, there is a PPT $\mathcal{S}$ that returns $(p, t)$ such that the commitment scheme $\Pi$ with the parameter $p$ is a trapdoor commitment scheme with trapdoor $t$ and the parameter generated by $\mathcal{S}$ is computationally indistinguishable from the parameter generated by $\left\langle P, M^{*}\right\rangle$.

It is worth noting that DPG-commitments are very different from the mixed commitments of [17]. For one thing, contrary to mixed commitments, DPG-commitments do not have equivocal parameters. Moreover, mixed commitments have parameters that with overwhelming probability become extractable based on a trapdoor hidden in the common reference string. In contrast, DPG-commitments become extractable due to the manipulation of the parameter generation protocol $M$ (specifically the ability of the 
simulator to rewind it). Now using the same arguments as in [28] it is possible to show that the commitment scheme in figure 3 is a UC-commitment provided that the underlying scheme used for $C_{1}$ is a DPG-commitment. We briefly sketch the proof argument. When the sender is corrupted, the simulator has to extract the committed message. This can be done by making $p S$ extractable. Then, given a commitment $\left\langle C_{1}, C_{2}, \pi\right\rangle$ from the adversary, the simulator can extract the message committed to from $C_{1}$ using the trapdoor of $p S$. When the receiver is corrupted, the simulator can make the commitment equivocal by causing $p R$ to be extractable. Using the trapdoor for $p R$ as witness, the simulator can generate a WI proofs $\pi$ and $\pi^{\prime}$ with respect to the condition (2) and thus open the commitment to an arbitrary message.

We next briefly argue that the construction suggested in [28] amounts to a DPGcommitment scheme. The token operates over a multiplicative cyclic group of prime order. In the first round, a party generates a cyclic group and sends to the token the group description and random elements $g$ and $h$ of the group; then, the token sends back a Pedersen commitment $c=\operatorname{com}\left(g_{1}, g_{2}\right)$ to random $g_{1}, g_{2}$ [36] 5 In the second final round, the party sends a random $h_{1}, h_{2}$, and then the token opens the commitment $c$ and outputs the signature on $\left(g, h, \hat{g_{1}}, \hat{g_{2}}\right)$ where $\hat{g_{1}}=g_{1} h_{1}$ and $\hat{g_{2}}=g_{2} h_{2}$. With parameter $\left(g, h, \hat{g_{1}}, \hat{g_{2}}\right)$, commitment $C_{1}$ to a bit $b$ is defined as $\left(g^{r_{1}} h^{r_{2}}, \hat{g}_{1}{ }^{r_{1}}{\hat{g_{2}}}^{r_{2}} g^{b}\right)$ for randomly-chosen $r_{1}, r_{2} \in \mathbb{Z}_{q}$. It is well-known (and easy to check) that if the parameter is a Diffie-Hellman (DH) tuple and $r=\log _{g} \hat{g_{1}}=\log _{h} \hat{g_{2}}$ is known, then $b$ can be efficiently extracted from the commitment. On the other hand, if it is a random tuple, this commitment scheme is perfectly hiding. Extraction mode is achieved by rewinding the code of a malicious token $M^{*}$. Specifically for a given $M^{*}$, the simulator $\mathcal{S}$ proceeds by picking a random DH tuple $\left(g, h, \hat{g_{1}}=g^{t}, \hat{g_{2}}=h^{t}\right)$ and running $M^{*}$ once to reach a successful termination and learn the values $g_{1}, g_{2}$. Subsequently, it rewinds $M^{*}$ right before the second round and selects $h_{1}=\hat{g_{1}} / g_{1}$ and $h_{2}=\hat{g_{2}} / g_{2}$. This will result in the parameter produced by $M^{*}$ to be equal to the DH tuple, i.e., a parameter that is extractable with trapdoor $t$.

\subsection{UC-Secure Commitment Scheme from a Tamperable Token}

It is easy to see that the following result holds using the BiTR security properties.

Corollary 4. If an ITM $M$, achieving parameters for DPG-commitment scheme, is $\mathcal{T}$ BiTR, then there exists a UC-secure commitment scheme in the $\mathcal{F}_{\text {twrap }}(M, \mathcal{T})$-hybrid model.

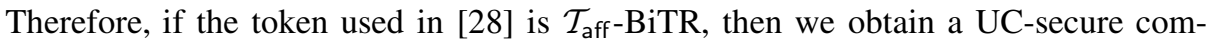
mitment scheme in the $\mathcal{F}_{\text {twrap }}\left(M, \mathcal{T}_{\text {aff }}\right)$-hybrid model. Unfortunately, the token is not

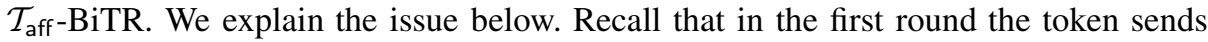
a commitment to $g_{1}, g_{2}$. Suppose that $g_{1}=g^{r_{1}}$ and $g_{2}=g^{r_{2}}$ and that the values $r_{1}$ and $r_{2}$ are stored as state in the token after the first round. Suppose in addition that by tampering with an affine function the adversary causes the state to become $\left(a r_{1}+b, r_{2}\right)$ for some $a$ and $b$. Then, in the second round, the simulator - given $h_{1}$ and $h_{2}$ from

\footnotetext{
${ }^{5}$ We use a slightly different notation compared to [28] to unify the presentation with our BiTR token that is shown later.
} 
Let $\mathbb{G}$ be the cyclic multiplicative group of size $q$ defined by a safe prime $p=2 q+1$ and $g$ be a generator of $\mathbb{G}$. The description of $\mathbb{G}$ is embedded in the program. The state is $\left(r_{1}, r_{2}, s_{1}, s_{2}\right) \in \mathbb{Z}_{q}^{4}$. It uses a signature ITM $K$ as a subprotocol.

\section{Initialization}

- Upon receiving a message (Initialize), call $K$ with (Initialize), sets the state to all 0 s and output whatever $K$ outputs.

\section{Message Handling}

- Upon receiving a message $h_{0}$ : Check $h_{0}$ is a generator of $\mathbb{G}$. If the checking fails, output $\perp$. Otherwise, pick $r_{i}, s_{i} \in_{R} \mathbb{Z}_{q}$ and compute Pedersen commitments $\operatorname{com}_{i}=g^{s_{i}} h_{0}^{\mathcal{X}\left(g_{i}\right)}$ for $i=1,2$, where $g_{i}=g^{r_{i}}$ and $\mathcal{X}$ is defined as: $\mathcal{X}(\alpha)=\alpha$ if $\alpha>p / 2, p-\alpha$ otherwise. Output $\left(\mathrm{com}_{1}, \mathrm{com}_{2}\right)$.

- Upon receiving a message $\left(h, h_{1}, h_{2}, x_{1}, x_{2}\right)$ : Check $h, h_{1}, h_{2} \in \mathbb{G}, x_{1}, x_{2} \in \mathbb{Z}_{q}^{*}$. If the checking fails, output $\perp$. Otherwise, let $g_{i}=g^{r_{i}}$ and compute $\hat{g}_{i}=g_{i}^{x_{i}} h_{i}$ for $i=1,2$. Call $K$ with $\left(\operatorname{Sign},\left(P, P^{\prime}, p, g, h, \hat{g}_{1}, \hat{g}_{2}\right)\right)$ to get a signature $\sigma$. Output $\left(g_{1}, g_{2}, s_{1}, s_{2}, \sigma\right)$. Pick $r_{i}, s_{i} \in \in_{R} \mathbb{Z}_{q}$ for $i=1,2$.

Fig. 4. Dual parameter generating ITM $M_{d p g}$ that is universal-BiTR parent

the adversary - has to send $\mathcal{F}_{\text {wrap }}$ appropriate messages $h_{1}^{\prime}$ and $h_{2}$ so that it can manipulate the output from $\mathcal{F}_{\text {wrap }}$ as if the result is from $\mathcal{F}_{\text {twrap }}$. Here the signature on $\left(g, h, \hat{g_{1}}, \hat{g_{2}}\right)$ is a critical obstacle, since the simulator cannot modify it (otherwise, it violates unforgeability of signature schemes). This means that for simulation to be successful it should hold that $\hat{g}_{1}=g^{a r_{1}+b} h_{1}=g^{r_{1}} h_{1}^{\prime}$, i.e., the simulator should select $h_{1}^{\prime}=g^{(a-1) r_{1}+b} h_{1}$. Unfortunately, the simulator does not know $r_{1}$ when it is supposed to send $h_{1}^{\prime}$.

By slightly changing the token above, however, we manage to obtain a DPG-achieving ITM $M_{d p g}$ that is BiTR against affine tampering functions. Its description is given in Fig. 4. First, we show $M_{d p g}$ achieves parameters for DPG-commitment. Roughly speaking, the protocol in the normal mode generates a random tuple $\left(g, h, \hat{g}_{1}, \hat{g}_{2}\right)$, by multiplying random numbers $g_{1}$ and $g_{2}$ (from $M_{d p g}$ ) and random numbers $h_{1}$ and $h_{2}$ (from the party). Therefore, the probability that the tuple $\left(g, h, \hat{g}_{1}, \hat{g}_{2}\right)$ is a DH tuple is negligible since $\hat{g}_{1}$ and $\hat{g}_{2}$ are uniformly distributed. In the extraction mode, however, the simulator emulating $\mathcal{F}_{\text {wrap }}$ can rewind the ITM to cause $\left(g, h, \hat{g}_{1}, \hat{g}_{2}\right)$ to be a DH tuple. Specifically, the simulator picks a random DH tuple $\left(g, h, \hat{g}_{1}, \hat{g}_{2}\right)$ and, after finding out the values $g_{1}, g_{2}$, rewinds the machine right before the second round and sends $h_{i}=\hat{g}_{i} / g_{i}^{x_{i}}$ for $i=1,2$. Under the DDH assumption, parameters from the normal mode and from the extraction mode are indistinguishable.

More importantly, $M_{d p g}$ is BiTR against affine tampering functions. To achieve BiTR security, we introduce $x_{1}$ and $x_{2}$. As before, suppose that the state for $g_{1}$ is changed from $r_{1}$ to $a r_{1}+b$. In the second round, the simulator - given $h_{1}$ and $x_{1}$ - has to send appropriate $h_{1}^{\prime}$ and $x_{1}^{\prime}$ to $\mathcal{F}_{w r a p}$ such that $\hat{g}_{1}=g^{\left(a r_{1}+b\right) x_{1}} h_{1}=g^{r_{1} x_{1}^{\prime}} h_{1}^{\prime}$. This means that $h_{1}^{\prime}=g^{z} h_{1}$ where $z=\left(a r_{1} x_{1}+b x_{1}-r_{1} x_{1}^{\prime}\right)$. The good news is that although the simulator doesn't know $r_{1}$, it does know how to pick $x_{1}^{\prime}$ to satisfy the equation: $x_{1}^{\prime}=a x_{1}$. The value $h_{1}^{\prime}$ can be computed subsequently from the above equation. 
Theorem 5. The ITM $M_{d p g}$ in Fig. 4 is $\mathcal{T}_{\text {aff }}^{4}$-BiTR.

Furthermore, the way the ITM $M_{d p g}$ uses a signature scheme is simple enough (it simply passes through whatever it receives from the signature token) and we can easily extend the above lemma to prove that $M_{d p g}$ is universal BiTR parent. We also show that the ITM for the Okamoto signature scheme $M_{o k a}$ is modular- $\mathcal{T}_{\text {aff }}-\mathrm{BiTR}$ when used with $M_{d p g}$.

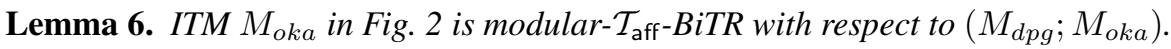

Applying the composition theorem (Theorem 1) along with Theorem 5 and Lemma 6 to the above scheme, we obtain a BiTR token that gives a UC commitment based on corollary 4.

Corollary 7. $\left(M_{d p g} ; M_{o k a}\right)$ is $\mathcal{T}_{\text {aff }}^{5}$-BiTR.

\section{BiTR Protocols against General Classes of Tampering Functions}

\subsection{BiTR Protocols from Non-malleable Codes}

In this section we will see how the BiTR property can be derived by implementing an integrity check in the form of an encoding $\psi$. A useful tool for this objective is the notion of non-malleable codes [21]. A pair of procedures $(E, D)$ is a non-malleable code with respect to tampering functions $\mathcal{T}$, if there is an algorithm $\mathcal{S}$ that detects whether the state becomes invalid, given only the tampering function $t$. In particular $\mathcal{S}$ should satisfy the following property: for all $x \in\{0,1\}^{n}$ and $t \in \mathcal{T}$, if $x=D(t(E(x)))$ (i.e., $x$ stays the same even after applying the tampering $t$ ), it holds that $\mathcal{S}(t)=\top$ with overwhelming probability, while otherwise $\mathcal{S}(t)$ is statistically (or computationally) close to $D(t(E(x)))$. By encoding the state of a protocol with a non-malleable code it is possible to show the following restatement of Theorem 6.1 of [21] under the BiTR security framework.

Theorem 8 ([21]). Let $\mathcal{T}$ be a class of tampering functions over $\{0,1\}^{m}$ and $(E, D, \mathcal{S})$ be a non-malleable code with respect to $\mathcal{T}$, where $E:\{0,1\}^{n} \rightarrow\{0,1\}^{m}, D$ : $\{0,1\}^{m} \rightarrow\{0,1\}^{n}$ and $\mathcal{S}$ are efficient procedures. Let $M$ be any ITM whose state is of length $n$. Then $M$ is $(\mathcal{T}, \psi)$-BiTR where $\psi=(E, D)$.

The above theorem suggests the importance of the problem of constructing non-malleable codes for a given class of tampering functions $\mathcal{T}$. Some positive answers to this difficult question are given in [21] for a class of tampering functions that operate on each one of the bits of the state independently; they also provide a general feasibility result for tampering families of bounded size (with an inefficient construction); an important characteristic of those solutions is relying on the randomness of the encoding. Here we show a different set of positive results by considering the case of deterministic nonmalleable codes, i.e., the setting where $(E, D)$ are both deterministic functions.

In our result we will utilize a relaxation of non-malleable codes: $(E, D$, Predict $)$ is called a $\delta$-non-malleable code with distance $\epsilon$ if for any $x \in\{0,1\}^{n}$ and $t \in \mathcal{T}$, it holds that (i) $D(E(x))=x$, (ii) the probability that $D(t(E(x)))$ is neither $x$ nor 
$\perp$ is at most $\delta 6$ and (iii) $\operatorname{Predict}(\cdot)$ outputs either $\top$ or $\perp$, and $\mid \operatorname{Pr}[D(t(E(x)))=$ $x]-\operatorname{Pr}[\operatorname{Predict}(t)=\top] \mid \leq \epsilon$. It is easy to see that if $\epsilon, \delta$ are negligible the resulting code is non-malleable: given that $\delta$ is negligible, property (ii) suggests that $D$ will return either the correct value or fail, and thus in case it fails, Predict $(\cdot)$ will return $\perp$ with about the same probability due to (iii). We call $\delta$ the crossover threshold and $\epsilon$ the predictability distance.

\subsection{Constructing Deterministic Non-malleable Codes}

Inefficient Construction for Any $\mathcal{T}$. We now consider the problem of constructing a $\delta$-non-malleable code $E:\{0,1\}^{n} \rightarrow\{0,1\}^{m}$ for a given class of tampering functions and parameters $\delta, \epsilon$. We will only consider the case when $\delta>\epsilon$ as the other case is not useful. We note that the construction is inefficient for large $m$ and $n$, but it becomes efficient for logarithmic values of $m, n$. Following this we utilize it in the construction of deterministic non-malleable codes.

For a given $t \in \mathcal{T}$ consider the graph $G$ that is defined with vertex set $V=\{0,1\}^{m}$ with each edge $\left(u_{1}, u_{2}\right)$ having weight $w_{t}\left(u_{1}, u_{2}\right)=\operatorname{Pr}\left[t\left(u_{1}\right)=u_{2}\right]{ }^{7}$ Finding a good $\delta$-non-malleable code amounts to finding a partition $S, \bar{S}=V \backslash S$ of $G$ satisfying the following properties that for each $t \in \mathcal{T}$ :

- For all $u, v \in S$, it holds that $w_{t}(u, v) \leq \delta$.

- Either (i) $\forall u \in S: \sum_{v \in \bar{S}} w_{t}(u, v) \geq 1-\epsilon$ or (ii) $\forall u \in S: \sum_{v \in \bar{S}} w_{t}(u, v) \leq \epsilon$.

If $S$ satisfies condition (i) (resp., condition (ii)) for a given $t \in \mathcal{T}$, we will say that $S$ is a repeller (resp., an attractor) with respect to $t$.

We next provide a simple algorithm that is guaranteed to produce a code of non-zero rate if such exists. Consider all pairs of vertices $\left\{u_{1}, u_{2}\right\}$ and classify them according to whether they are repellers or attractors with parameters $\delta, \epsilon$. Note that testing whether these sets are repellers or attractors requires $O(|V|)$ steps. We perform the same for all tampering functions $t \in \mathcal{T}$ and then consider only those sets that appear in the list of all tampering functions. Finally, we improve the size of such a selected pair by moving vertices from $\bar{S}$ to $S$ provided that the repeller or attractor property is maintained. We note that this approach will enable us to reach a local maximum code nevertheless it is not guaranteed to find an optimal code.

Assume now that the output of the above procedure is the set $\mathcal{C} \subseteq V=\{0,1\}^{m}$. We next set $n=\left\lfloor\log _{2}|\mathcal{C}|\right\rfloor$ and consider $E:\{0,1\}^{n} \rightarrow\{0,1\}^{m}$ an arbitrary injection from $\{0,1\}^{n}$ to $\mathcal{C}$. The decoding $D$ is defined as the inverse of $E$ when restricted on $\mathcal{C}$, and $\perp$ everywhere else. We next define Predict as follows. On input $t$, if $\mathcal{C}$ is an attractor, then output ok; otherwise output $\perp$ (i.e., for the case $\mathcal{C}$ is an repeller).

\footnotetext{
${ }^{6}$ The tampering $t$ may change the codeword $x$ into another valid codeword.

${ }^{7}$ In the above description, we assumed the probabilities $\operatorname{Pr}[t(c)=u]$ are known. If they are not known, they can be estimated using standard techniques. In particular, to evaluate the probability of an event $A$, repeat $k$ independent experiments of $A$ and denote the success ratio of the $k$ experiments as $\hat{p}$. Let $X_{i}$ be the probability that the $i$-th execution of the event $A$ is successful. The expected value of $Y=\sum_{i=1}^{k} X_{i}$ is $k \cdot p$. Using the Chernoff bound it follows that $|\hat{p}-p| \leq 1 / N$ with probability $1-\gamma$ provided that $k=\Omega\left(N^{2} \ln \left(\gamma^{-1}\right)\right)$.
} 
The rate of the constructed code is $n / m$, while the time-complexity of constructing $E, D$, Predict $(\cdot)$ is $2^{\mathcal{O}(n)}|\mathcal{T}|$. The size of the circuit evaluating each one of these functions is respectively $2^{n}, 2^{m},|\mathcal{T}|$.

Theorem 9. Fix any class of functions $\mathcal{T}$. If there exists a code (E,D, Predict) with rate $>0$ that is $\delta$-non-malleable w.r.t. $\mathcal{T}$ and distance $\epsilon$, then such a code is produced by the above procedure.

When does a deterministic non-malleable code exist? The basic idea of the construction above was to search for a one-sided set of codewords and use it to define the non-malleable code. The necessity of one-sidedness is easy to see since if the property fails, i.e., $\epsilon<q_{u, t}<1-\epsilon$ for some $t$ and $u$, the requirement on Predict cannot hold in general since it cannot predict with high probability what would happen in the real world after tampering a state that is encoded as $u$. We now provide two illustrative examples and discuss the existence (and rate) of a deterministic non-malleable encoding for them.

Example 1: Set Functions. If $\mathcal{T}$ contains a function $t$ that sets the $i$-th bit of $u \in\{0,1\}^{m}$ to 0 , it follows that the code $\mathcal{C}$ we construct must obey that either all codewords have the $i$-th bit set to 0 or all of them have the bit set to 1 . This means that the inclusion of any bit setting function in $\mathcal{T}$ cuts the size of the code $|\mathcal{C}|$ by half. There is no nonmalleable code when the collection $\mathcal{T}$ contains Set functions for every bit position (this is consistent with the impossibility result of [23] for algorithmic tamper proof security when Set functions are allowed for tampering).

Example 2: Differential Fault Analysis [8]. Consider a single function $t$ which flips each 1-bit to a 0-bit with probability $\beta$. Consider a code $\mathcal{C} \subseteq\{0,1\}^{m}$ for which it holds that all codewords in $\mathcal{C}$ have Hamming distance at least $r$ between each other and $0^{m} \in \mathcal{C}$. Then it is easy to see that $\delta$, the probability of crossover, is at most $\beta^{r}$. Further, now suppose that $t$ is applied to an arbitrary codeword $u$ in $\mathcal{C}$ other than $0^{m}$. We observe that the number of 1's in $u$ is at least $r$ (otherwise it would have been too close to $0^{m}$ ). It follows that $t$ will change some of these 1 's to 0 's, with probability at least $1-(1-\beta)^{r}$. It follows that we can predict the effect of the application of $t$ with this probability when we restrict to codewords in $\mathcal{C} \backslash\left\{0^{m}\right\}$. In summary, any code $\mathcal{C}$ over $\{0,1\}^{m}$ with minimum distance $r$ that contains $0^{m}$ allows for a $\beta^{r}$-non-malleable code with $(1-\beta)^{r}$ for $t$ using the code $\mathcal{C} \backslash\left\{0^{m}\right\}$.

We can extend the above to the case when $a$ compositions of $t$ are allowed. Note that a sequence of $a$ applications of $t$ will flip each 1-bit to a 0-bit with probability $\beta+(1-\beta) \beta+\ldots+(1-\beta)^{a-1} \beta=1-(1-\beta)^{a}$. The encoding now has crossover $\left(1-(1-\beta)^{a}\right)^{r} \leq e^{-(1-\beta)^{a} r}$. Thus, from $e^{-(1-\beta)^{a} r} \leq \delta$, we obtain $r \geq(1 /(1-$ $\beta)^{a} \ln (1 / \delta)$, i.e., when $\beta$ is bounded away from 1 , the minimum distance of the code grows exponentially with $a$.

Efficient Construction for Localized $\mathcal{T}$. Now, we show a simple way to use the (inefficient) construction of the beginning of the section with constant rate and any cross-over $\delta<1 / 2$, to achieve an efficient construction with negligible cross-over (and thus, BiTR security for any protocol $M$ whose state is encoded with the resulting code), when the class contains only functions that can be split into independent tampering 
of local (i.e., logarithmically small) blocks. Here we consider a tampering class $\mathcal{T}$ of polynomial size. Roughly speaking, the construction is achieved first by applying a Reed-Solomon code to the overall state and then by applying the $\delta$-non-malleable code to the resulting codeword in small blocks. Let $\mathcal{T}^{\ell}$ denote $\mathcal{T} \times \cdots \times \mathcal{T}$ (with $\ell$ repetitions).

Theorem 10. Let $k$ be a security parameter. Let $\mathcal{T}$ be a class of functions over $\{0,1\}^{m}$ with $m=\mathcal{O}(\log k)$ for which a $\delta$-non-malleable code exists and is efficiently constructible with rate $r$. Then there is an efficiently constructible deterministic non-malleable code w.r.t. $\mathcal{T}^{\ell}$ for any rate less than $(1-\delta) r$ provided $\ell / \log \ell=\omega(\log k)$.

Acknowledgement. We are grateful to Li-Yang Tan and Daniel Wichs for useful discussions regarding this work.

\section{References}

1. Alwen, J., Dodis, Y., Wichs, D.: Leakage-Resilient Public-Key Cryptography in the BoundedRetrieval Model. In: Halevi, S. (ed.) CRYPTO 2009. LNCS, vol. 5677, pp. 36-54. Springer, Heidelberg (2009)

2. Anderson, R.J., Kuhn, M.G.: Tamper resistance - a cautionary note. In: The Second USENIX Workshop on Electronic Commerce Proceedings, Oakland, California, pp. 1-11 (18-21, 1996)

3. Applebaum, B., Harnik, D., Ishai, Y.: Semantic security under related-key attacks and applications. In: Innovations in Computer Science - ICS 2011, pp. 45-60 (2011)

4. Bar-El, H., Choukri, H., Naccache, D., Tunstall, M., Whelan, C.: The sorcerers apprentice guide to fault attacks. Cryptology ePrint Archive, Report 2004/100 (2004), http://eprint.iacr.org/

5. Bellare, M., Cash, D.: Pseudorandom Functions and Permutations Provably Secure against Related-Key Attacks. In: Rabin, T. (ed.) CRYPTO 2010. LNCS, vol. 6223, pp. 666-684. Springer, Heidelberg (2010)

6. Bellare, M., Kohno, T.: A Theoretical Treatment of Related-Key Attacks: Rka-prps, Rka-prfs, and Applications. In: Biham, E. (ed.) EUROCRYPT 2003. LNCS, vol. 2656, pp. 491-506. Springer, Heidelberg (2003)

7. Biham, E., Shamir, A.: Differential Fault Analysis of Secret Key Cryptosystems. In: Kaliski Jr., B.S. (ed.) CRYPTO 1997. LNCS, vol. 1294, pp. 513-525. Springer, Heidelberg (1997)

8. Boneh, D., DeMillo, R.A., Lipton, R.J.: On the importance of eliminating errors in cryptographic computations. J. Cryptology 14(2), 101-119 (2001)

9. Boyle, E., Segev, G., Wichs, D.: Fully Leakage-Resilient Signatures. In: Paterson, K.G. (ed.) EUROCRYPT 2011. LNCS, vol. 6632, pp. 89-108. Springer, Heidelberg (2011)

10. Brakerski, Z., Kalai, Y.T., Katz, J., Vaikuntanathan, V.: Overcoming the hole in the bucket: Public-key cryptography resilient to continual memory leakage. In: FOCS, pp. 501-510 (2010)

11. Brumley, D., Boneh, D.: Remote timing attacks are practical. Computer Networks 48(5), 701-716 (2005)

12. Canetti, R.: Universally composable security: A new paradigm for cryptographic protocols. In: FOCS, pp. 136-145 (2001)

13. Canetti, R., Fischlin, M.: Universally Composable Commitments. In: Kilian, J. (ed.) CRYPTO 2001. LNCS, vol. 2139, pp. 19-40. Springer, Heidelberg (2001)

14. Canetti, R., Goldreich, O., Goldwasser, S., Micali, S.: Resettable zero-knowledge (extended abstract). In: STOC, pp. 235-244 (2000) 
15. Canetti, R., Kushilevitz, E., Lindell, Y.: On the Limitations of Universally Composable TwoParty Computation Without Set-Up Assumptions. In: Biham, E. (ed.) EUROCRYPT 2003. LNCS, vol. 2656, pp. 68-86. Springer, Heidelberg (2003)

16. Chandran, N., Goyal, V., Sahai, A.: New Constructions for UC Secure Computation Using Tamper-Proof Hardware. In: Smart, N.P. (ed.) EUROCRYPT 2008. LNCS, vol. 4965, pp. 545-562. Springer, Heidelberg (2008)

17. Damgård, I., Nielsen, J.B.: Perfect Hiding and Perfect Binding Universally Composable Commitment Schemes with Constant Expansion Factor. In: Yung, M. (ed.) CRYPTO 2002. LNCS, vol. 2442, pp. 581-596. Springer, Heidelberg (2002)

18. Damgård, I., Nielsen, J.B., Wichs, D.: Isolated Proofs of Knowledge and Isolated Zero Knowledge. In: Smart, N.P. (ed.) EUROCRYPT 2008. LNCS, vol. 4965, pp. 509-526. Springer, Heidelberg (2008)

19. Dodis, Y., Haralambiev, K., López-Alt, A., Wichs, D.: Cryptography against continuous memory attacks. In: FOCS, pp. 511-520 (2010)

20. Döttling, N., Kraschewski, D., Müller-Quade, J.: Unconditional and Composable Security Using a Single Stateful Tamper-Proof Hardware Token. In: Ishai, Y. (ed.) TCC 2011. LNCS, vol. 6597, pp. 164-181. Springer, Heidelberg (2011)

21. Dziembowski, S., Pietrzak, K., Wichs, D.: Non-malleable codes. In: ICS, pp. 434-452 (2010)

22. Faust, S., Kiltz, E., Pietrzak, K., Rothblum, G.N.: Leakage-Resilient Signatures. In: Micciancio, D. (ed.) TCC 2010. LNCS, vol. 5978, pp. 343-360. Springer, Heidelberg (2010)

23. Gennaro, R., Lysyanskaya, A., Malkin, T., Micali, S., Rabin, T.: Algorithmic Tamper-Proof (ATP) Security: Theoretical Foundations for Security against Hardware Tampering. In: Naor, M. (ed.) TCC 2004. LNCS, vol. 2951, pp. 258-277. Springer, Heidelberg (2004)

24. Goyal, V., Ishai, Y., Mahmoody, M., Sahai, A.: Interactive Locking, Zero-Knowledge PCPs, and Unconditional Cryptography. In: Rabin, T. (ed.) CRYPTO 2010. LNCS, vol. 6223, pp. 173-190. Springer, Heidelberg (2010)

25. Goyal, V., Ishai, Y., Sahai, A., Venkatesan, R., Wadia, A.: Founding Cryptography on Tamper-Proof Hardware Tokens. In: Micciancio, D. (ed.) TCC 2010. LNCS, vol. 5978, pp. 308-326. Springer, Heidelberg (2010)

26. Ishai, Y., Prabhakaran, M., Sahai, A., Wagner, D.: Private Circuits II: Keeping Secrets in Tamperable Circuits. In: Vaudenay, S. (ed.) EUROCRYPT 2006. LNCS, vol. 4004, pp. 308-327. Springer, Heidelberg (2006)

27. Ishai, Y., Sahai, A., Wagner, D.: Private Circuits: Securing Hardware against Probing Attacks. In: Boneh, D. (ed.) CRYPTO 2003. LNCS, vol. 2729, pp. 463-481. Springer, Heidelberg (2003)

28. Katz, J.: Universally Composable Multi-party Computation Using Tamper-Proof Hardware. In: Naor, M. (ed.) EUROCRYPT 2007. LNCS, vol. 4515, pp. 115-128. Springer, Heidelberg (2007)

29. Kocher, P.C., Jaffe, J., Jun, B.: Differential Power Analysis. In: Wiener, M. (ed.) CRYPTO 1999. LNCS, vol. 1666, pp. 388-397. Springer, Heidelberg (1999)

30. Kolesnikov, V.: Truly Efficient String Oblivious Transfer Using Resettable Tamper-Proof Tokens. In: Micciancio, D. (ed.) TCC 2010. LNCS, vol. 5978, pp. 327-342. Springer, Heidelberg (2010)

31. Lewko, A.B., Lewko, M., Waters, B.: How to leak on key updates. In: STOC, pp. 725-734 (2011)

32. Malkin, T., Teranishi, I., Vahlis, Y., Yung, M.: Signatures Resilient to Continual Leakage on Memory and Computation. In: Ishai, Y. (ed.) TCC 2011. LNCS, vol. 6597, pp. 89-106. Springer, Heidelberg (2011)

33. Micali, S., Reyzin, L.: Physically Observable Cryptography (Extended Abstract). In: Naor, M. (ed.) TCC 2004. LNCS, vol. 2951, pp. 278-296. Springer, Heidelberg (2004) 
34. Moran, T., Segev, G.: David and Goliath Commitments: UC Computation for Asymmetric Parties Using Tamper-Proof Hardware. In: Smart, N.P. (ed.) EUROCRYPT 2008. LNCS, vol. 4965, pp. 527-544. Springer, Heidelberg (2008)

35. Okamoto, T.: Efficient Blind and Partially Blind Signatures Without Random Oracles. In: Halevi, S., Rabin, T. (eds.) TCC 2006. LNCS, vol. 3876, pp. 80-99. Springer, Heidelberg (2006)

36. Pedersen, T.P.: Non-interactive and Information-Theoretic Secure Verifiable Secret Sharing. In: Feigenbaum, J. (ed.) CRYPTO 1991. LNCS, vol. 576, pp. 129-140. Springer, Heidelberg (1992)

37. Pietrzak, K.: A Leakage-Resilient Mode of Operation. In: Joux, A. (ed.) EUROCRYPT 2009. LNCS, vol. 5479, pp. 462-482. Springer, Heidelberg (2009)

38. Quisquater, J.J., Samyde, D.: ElectroMagnetic Analysis (EMA): Measures and CounterMeasures for Smart Cards. In: Attali, S., Jensen, T. (eds.) E-smart 2001. LNCS, vol. 2140, pp. 200-210. Springer, Heidelberg (2001)

39. Schnorr, C.P.: Efficient signature generation by smart cards. J. Cryptology 4(3), 161-174 (1991)

40. Skorobogatov, S.P.: Semi-invasive attacks - A new approach to hardware security analysis. Tech. Rep. UCAM-CL-TR-630, University of Cambridge, Computer Laboratory (April 2005), http: / /www.cl.cam.ac.uk/techreports/UCAM-CL-TR-630.pdf

41. Standaert, F.X., Malkin, T.G., Yung, M.: A Unified Framework for the Analysis of SideChannel Key Recovery Attacks. In: Joux, A. (ed.) EUROCRYPT 2009. LNCS, vol. 5479, pp. 443-461. Springer, Heidelberg (2009) 